\title{
Neutrinoless double beta decay in Bilinear R-parity breaking SUSY
}

\author{
M. Hirsch * \\ Instituto de Física Corpuscular - C.S.I.C. \\ Departamento de Física Teòrica, \\ Universidad de València \\ 46100 Burjassot, València \\ E-mail: 'mahirsch@flamenco.ific.uv.es'
}

AbstRACT: Supersymmetric models with broken R-parity generate Majorana masses for neutrinos. Since neutrinoless double beta decay $\left(\beta \beta_{0 \nu}\right)$ is a $\Delta L=2$ process, it is especially sensitive to the Majorana nature of the neutrino and from the non-observation of this process stringent limits for $\mathrm{R}$-parity violating parameters of the first generation can be derived. Here, $\beta \beta_{0 \nu}$ decay is studied in models where R-parity is broken via bilinear terms. Generally it is found that, although there are a number of diagrams which contribute at the same level of perturbation theory the usual mass mechanism of $\beta \beta_{0 \nu}$ decay is the dominant contribution. Non-observation of $\beta \beta_{0 \nu}$ decay then gives upper limits on R-parity violating parameters of the order of typically a (few) $100 \mathrm{keV}$ to a (few) $\mathrm{MeV}$.

\section{Introduction}

The minimal supersymmetric extension of the standard model (MSSM) [1,in] contains a symmetry called R-parity, defined as $R_{P}=(-1)^{3 B+L+2 S}$, where $B$ and $L$ are the baryon and lepton number and $S$ the spin of the corresponding particle. However, theoretically R-parity is not well motivated and it has been realized already years ago that the violation of R-parity can arise as residual effects in larger theories [2] to a sneutrino vacuum expectation value (VEV) [3יin].

R-parity violating (RPV) realizations of supersymmetry have become increasingly interesting, especially from the phenomenological point of view, due to the mounting experimental evi-

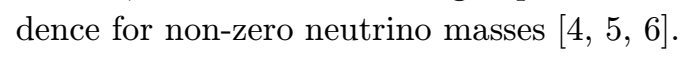

RPV models allow, without any fine-tuning of parameters, for relatively small - and in principle calculable - Majorana neutrino masses. Since Majorana neutrino masses violate necessarily lep-

\footnotetext{
* based on work done in collaboration with J.W.F. Valle, hep-ph/9812463
}

ton number by two units, one expects that neutrinoless double beta decay, being a $\Delta L=2$ process too, must also occur if RPV SUSY is realized in nature. ${ }^{1}$

Unfortunately, $\beta \beta_{0 \nu}$ decay has yet to be observed, but its absence allows to put stringent constraints on RPV supersymmetry. This has been shown for models with explicit trilinear Rparity breaking in $\left[{ }_{1}^{1}, \overline{9}\right]$, and for bilinear R-parity breaking in $\left[\begin{array}{ll}1 \\ 1\end{array} 0_{n}^{1} 111_{1}^{1}\right.$. This talk is mainly based on [11i]. For a discussion of the differences between

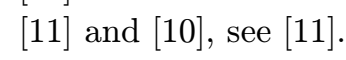

\section{Supersymmetry with bilinear R- parity breaking}

In the presence of bilinear R-parity and lepton number violation there is no distinction between the lepton doublet and the Higgs doublet superfield giving mass to the down-type quarks. This

\footnotetext{
${ }^{1}$ The connection between Majorana neutrino masses and $\beta \beta_{0 \nu}$ decay is quite general $\left[r_{1}\right]$ and not restricted to RPV SUSY.
} 
fact can be accounted for by defining a superfield $\hat{\Phi}$ as

$$
\hat{\Phi}=\left(\hat{H}_{1}, \hat{L}_{1}, \hat{L}_{2}, \hat{L}_{3}\right) .
$$

For the MSSM field content the most general gauge invariant form of the renormalizable superpotential can then be written as

$$
\begin{aligned}
W= & \epsilon_{a b}\left[\lambda_{e}^{I J k} \hat{\Phi}_{I}^{a} \hat{\Phi}_{J}^{b} \hat{E}_{k}^{C}+\lambda_{d}^{I j k} \hat{\Phi}_{I}^{a} \hat{Q}_{j}^{b} \hat{D}_{k}^{C}\right. \\
& \left.+h_{u}^{j k} \hat{Q}_{j}^{a} \hat{H}_{2}^{b} \hat{U}_{k}^{C}+\mu^{I} \hat{\Phi}_{I}^{a} \hat{H}_{2}^{b}\right] .
\end{aligned}
$$

Here, $\hat{Q}$ and $\hat{D}^{C}, \hat{U}^{C}$ are the quark doublet and singlets superfields, respectively, $\hat{E}^{C}$ is the lepton singlet superfield and $\hat{H}_{2}$ the Higgs superfields with $Y\left(\hat{H}_{2}\right)=1$ responsible for the up-type quark masses, with $h_{u}^{j k}$ being the corresponding Yukawa couplings. The indices $j, k=1,2,3$ denote generations, whereas $I, J=0,1,2,3$. The indices $a, b$ are $S U(2)$ indices. In the basis (2.11) one can separate $W$ into an R-parity conserving and an R-parity violating $\left(\not R_{p}\right)$ part

$$
W=W_{R_{P}}+W_{R_{p}},
$$

where the $R_{P}$ part is simply given by taking $I$ or $J$ to be zero and $i, j$ respectively. Then $\lambda_{e}^{0 j k}=$ $\frac{1}{2} h_{u}^{j k}$ and $\lambda_{d}^{0 j k}=h_{d}^{j k}$. The $R_{p}$ part contains the remaining components,

$$
W_{R_{p}}=\lambda_{e}^{i j k} \hat{L}_{i} \hat{L}_{j} \hat{E}_{k}^{C}+\lambda_{d}^{i j k} \hat{L}_{i} \hat{Q}_{j} \hat{D}_{k}^{C}+\epsilon_{i} \hat{L}_{i} \hat{H}_{2}
$$

where $\mu_{i} \rightarrow \epsilon_{i}$, to make contact with the notation of [12 2]. If $W_{R_{p}}$ would be the only source of RPV one could easily rotate the field $\hat{\Phi}$ into a basis $\hat{\Phi}^{\prime}$ with $\mu^{I^{\prime}}=\left(\mu^{\prime}, 0,0,0\right)$ effectively eliminating the bilinear terms. However, another source of RPV is found in the soft supersymmetry breaking part of the scalar potential. It contains the terms:

$$
\begin{aligned}
V_{R_{p}}^{\text {soft }} & =\tilde{A}_{e}^{I J k} \tilde{\Phi}_{I} \tilde{\Phi}_{J} \tilde{E}_{k}^{C}+\tilde{A}_{d}^{I j k} \tilde{\Phi}_{I} \tilde{Q}_{j} \tilde{D}_{k}^{C}+B^{I} \tilde{\Phi}_{I} H_{2} \\
& +\left(m_{I J}^{2}+\mu_{I} \mu_{J}\right) \tilde{\Phi}_{I} \tilde{\Phi}_{J}^{\dagger}+\cdots+\text { h.c. },
\end{aligned}
$$

where the dots represent terms not interesting for the discussion here. Rotating the superpotential as discussed above, it is easy to see that as long as the $B^{I}$ are not exactly parallel to the $\mu^{I}$ the effects of the bilinear terms of the superpotential in the rotated basis will reappear in the soft
SUSY breaking terms. For this reason the bilinear terms are physical and have to be taken into account in all RPV SUSY models. ${ }^{2}$

The presence of the lepton number violating bilinear terms in the superpotential, see eq. (2.3), leads to mixing of the neutralino and neutrino states. The full neutrino-neutralino mass matrix, for 3 generations of neutrinos can be written in the following form,

$$
\mathcal{M}_{0}=\left(\begin{array}{cc}
0 & m \\
m^{T} & \mathcal{M}_{\chi^{0}}
\end{array}\right) .
$$

Here, the submatrix $m$ contains entries from the bilinear $R_{p}$ parameters,

$$
m=\left(\begin{array}{cccc}
-\frac{1}{2} g^{\prime} \omega_{e} & \frac{1}{2} g \omega_{e} & 0 & -\epsilon_{e} \\
-\frac{1}{2} g^{\prime} \omega_{\mu} & \frac{1}{2} g \omega_{\mu} & 0 & -\epsilon_{\mu} \\
-\frac{1}{2} g^{\prime} \omega_{\tau} & \frac{1}{2} g \omega_{\tau} & 0 & -\epsilon_{\tau}
\end{array}\right),
$$

where the $\omega_{i}$ stand for the sneutrino vevs, $\omega_{i}:=$ $\left\langle\tilde{\nu}_{i}\right\rangle . \mathcal{M}_{\chi^{0}}$ is the MSSM neutralino mass matrix, given by,

$$
\mathcal{M}_{\chi^{0}}=\left(\begin{array}{cccc}
M_{1} & 0 & -\frac{1}{2} g^{\prime} v_{1} & \frac{1}{2} g^{\prime} v_{2} \\
0 & M_{2} & \frac{1}{2} g v_{1} & -\frac{1}{2} g v_{2} \\
-\frac{1}{2} g^{\prime} v_{1} & \frac{1}{2} g v_{1} & 0 & -\mu \\
\frac{1}{2} g^{\prime} v_{2} & -\frac{1}{2} g v_{2} & -\mu & 0
\end{array}\right) .
$$

Diagonalizing the mass matrix $(\overline{2} . \overline{6})$ one obtains seven mass eigenstates, from which at tree-level two remain exactly massless, but mixed with the other states.

If the $R_{p}$ parameters are small in the sense that for

$$
\xi=m \cdot \mathcal{M}_{\chi^{0}}^{-1}
$$

all $\xi_{i j} \ll 1$, one can find an approximate solution for the neutrino/neutralino mass matrix [i1 1 1 . Although (2.9i) need not to be true a priori, it is convenient to assume and justify it a posteriori. In this limit it is easy to see, why two neutrinos remain massless at tree-level, while the remaining neutrino mass is approximately given by

$$
m_{\nu}=\frac{M_{1} g^{2}+M_{2}{g^{\prime}}^{2}}{4 \operatorname{det}\left(\mathcal{M}_{\chi^{0}}\right)}|\vec{\Lambda}|^{2} .
$$

${ }^{2}$ The following discussion will mainly concentrate on the influence of the bilinear terms on $\beta \beta_{0 \nu}$ decay, for a discussion of $\beta \beta_{0 \nu}$ decay and trilinear RPV see [ [8, '92 ' 9'. 
Here, $|\vec{\Lambda}|$ is defined as

$$
\Lambda_{i}=\mu \omega_{i}-v_{1} \epsilon_{i}
$$

and contains all relevant information about the bilinear R-parity violation at tree-level.

In order to calculate the contributions to $\beta \beta_{0 \nu}$ decay, one has to find all possible lepton number violating interactions, involving the first generation, for the model under consideration. For the bilinear RPV model the relevant terms are [1] i ${ }_{1}$,

$$
\begin{aligned}
\mathcal{L}_{R_{p}}^{(\text {Bilinear })}= & -\frac{g}{\sqrt{2}} \kappa_{n} W_{\mu}^{-}\left(\bar{e} \gamma^{\mu} P_{L} \chi_{n}^{0}\right)+ \\
& \sqrt{2} g\left\{\beta_{k}^{u}\left(\bar{\nu}_{k} P_{R} u^{C}\right) \tilde{u}_{L}\right. \\
& +\beta_{k}^{d}\left(\bar{\nu}_{k} P_{R} d\right) \tilde{d}_{R}^{*} \\
& +\beta_{k i}^{e}\left(\bar{\nu}_{k} P_{R} e^{C}\right) \tilde{e}_{L i} \\
& \left.+\beta^{c}\left(\bar{u} P_{R} e^{C}\right) \tilde{d}_{L}\right\}+ \text { h.c. }
\end{aligned}
$$

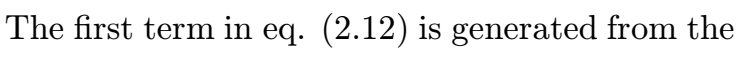
Standard Model $W$-boson-fermion-fermion and R-parity-conserving interactions of the type $W-$ $\chi^{0}-\chi^{ \pm}$present in the MSSM. The R-parityviolating terms arise from the $\chi^{ \pm}$-fermion-sfermion and $\chi^{0}$-fermion-sfermion interactions.

The coefficients in eq. (12.1 $\overline{1}$ ) are defined as follows,

$$
\begin{gathered}
\kappa_{n}=\sum_{k=1}^{3} \Delta_{1 k}^{-} \Xi_{n+3, k}^{*}+\sqrt{2} \Delta_{14}^{-} \Xi_{n+3,5}^{*}+\Delta_{15}^{-} \Xi_{n+3,6}^{*} \\
\beta_{k}^{d}=-\frac{1}{3} \tan \theta_{W} \Xi_{k, 4} \\
\beta_{k}^{u}=-\frac{1}{6}\left(\tan \theta_{W} \Xi_{k, 4}+3 \Xi_{k, 5}\right) \\
\beta_{k i}^{e}=-\frac{1}{\sqrt{2}} \Delta_{14}^{-} \Xi_{i, k}+\frac{1}{2}\left(\tan \theta_{W} \Xi_{k, 4}+\Xi_{k, 5}\right) \delta_{i 1} \\
\beta^{c}=-\frac{1}{\sqrt{2}} \Delta_{14}^{-} .
\end{gathered}
$$

Indices $i, k$ above run from 1 to 3 , whereas the index $n=1,2,3,4$. Here, $\Delta^{-}$and $\Xi$ are the matrices which diagonalize the charged leptonchargino and neutrino-neutralino mass matrices, for details see $\left[1 \overline{1} \overline{1}_{1}\right.$.

Neutrinoless double beta decay in this model occurs through two different sources: a) The finite neutrino mass, see ('-10.10) and b) through the finite neutrino-neutralino as well as the chargino-

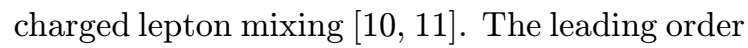
diagrams are summarized in figure 1.
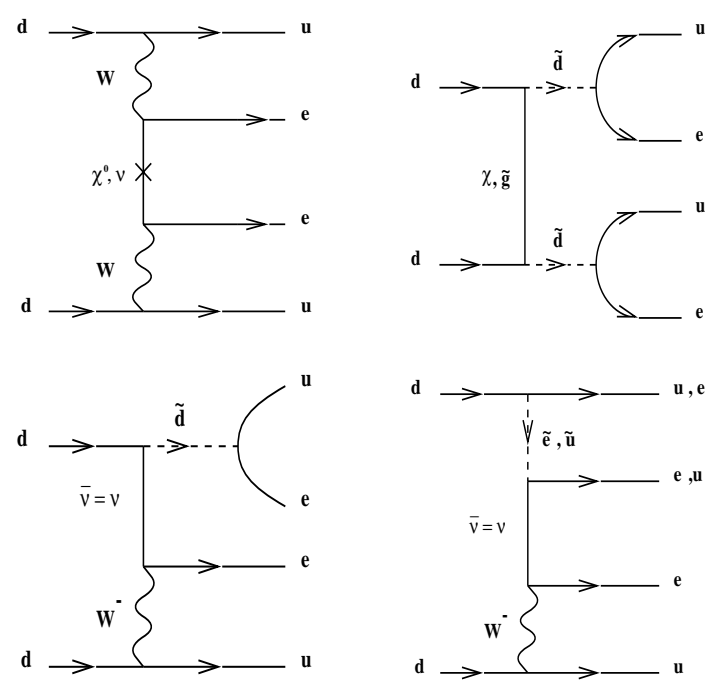

Figure 1: Leading double-beta-decay Feynman graphs in the $\not R_{p}$ model.

Figure 1.a (top left) shows the ordinary mass mechanism of $\beta \beta_{0 \nu}$ decay, accompanied by heavy neutralino exchange, fig. 1.b (top right) shows the gluino/neutralino exchange contribution, which is due to the non-zero charged-lepton-chargino mixing in this model. Figures 1.c and 1.d are neutrino-like graphs accompanied by supersymmetric particle exchange. Such vector-scalar graphs gave quite interesting constraints in models with trilinear R-parity breaking [i $\overline{1} \overline{3}_{1}$. In the bilinear model, however, they give non-zero contributions only in combination with trilinear RPV parameters, or suppressed by Yukawa couplings. Thus, although fig 1.c and 1.d are of the same order in perturbation theory as fig. 1.a they can be safely neglected in the analysis.

The half life for $\beta \beta_{0 \nu}$ decay can then be written as

$$
\left(T_{1 / 2}^{\beta \beta_{0 \nu}}\right)^{-1}=G_{01}\left(\mathcal{M}_{\nu}\right)^{2}\left|\eta^{\Delta L=2}\right|^{2} .
$$

Here, $G_{01}$ is the leptonic phase space integral, numerical values can be found in [1] nuclear matrix element governing the well-known mass mechanism of double beta decay and 


\section{Discussion of the results}

$$
\eta^{\Delta L=2}=\frac{\left\langle m_{\nu}\right\rangle}{m_{e}}+\left(\frac{m_{P}}{\left\langle m_{\chi}\right\rangle}+\eta_{\tilde{g}}\right) \mathcal{R}_{N}
$$

where $\mathcal{R}_{N}=\mathcal{M}_{N} / \mathcal{M}_{\nu}$, and the vector-scalar graphs have been neglected. The effective Majorana neutrino mass, $\left\langle m_{\nu}\right\rangle$, the effective neutralino mass $\left\langle m_{\chi}\right\rangle$ and $\eta_{\tilde{g}}$ are defined by,

$$
\begin{gathered}
\left\langle m_{\nu}\right\rangle=\sum_{j}^{\prime} U_{e j}^{2} m_{j} \simeq \frac{2}{3} \frac{g^{2} M_{2}}{\operatorname{det}\left(\mathcal{M}_{\chi^{0}}\right)} \Lambda_{1}^{2}, \\
\eta_{\chi}=\sum_{i=1}^{4} \frac{m_{p}}{m_{\chi_{i}^{0}}} \kappa_{i}^{2}=: \frac{m_{p}}{\left\langle m_{\chi}\right\rangle}, \\
\eta_{\tilde{g}}=\left(\frac{4 \pi \alpha_{S}}{9}\right)\left(\frac{4 \pi \alpha_{2}}{G_{F}^{2} m_{\tilde{d}_{L}}^{4}}\right)\left(\frac{m_{p}}{m_{\tilde{g}}}\right)\left(\beta^{c}\right)^{2},
\end{gathered}
$$

where $G_{F}$ is the Fermi constant, $m_{p}$ is the proton mass and $m_{\tilde{g}, \tilde{d}_{L}}$ the gluino, dsquark mass. The final results do not depend on the numerical values of $m_{e}$ and $m_{p}$ which appear in these definitions, since compensating factors appear in the original definitions for the nuclear matrix elements

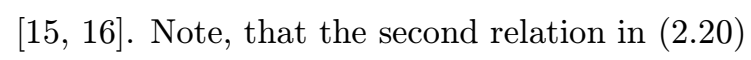
has been derived assuming $\Lambda_{1}$ to be smaller than the typical SUSY scale and using the usual GUT assumption on $M_{1}, M_{1}=(5 / 3) \tan ^{2} \theta_{W} M_{2}$.

Individual nuclear matrix elements are defined by,

$$
\begin{aligned}
& \mathcal{M}_{\nu}=\left(M_{G T, m}-\left(\frac{g_{V}}{g_{A}}\right)^{2} M_{F, m}\right), \\
& \mathcal{M}_{N}=\left(M_{G T, N}-\left(\frac{g_{V}}{g_{A}}\right)^{2} M_{F, N}\right),
\end{aligned}
$$

numerical values can be found in the literature. In the following analysis the matrix elements of $[1-1,1,16]$ have been used. Applying different calculations of the nuclear structure matrix elements lead to slightly different bounds. However, given the large margin of freedom in the MSSM parameters $\mu, M_{2}$ and $\tan (\beta)$, and the rather weak dependence of the limits on RPV parameters on the nuclear matrix elements $\left(\Delta \Lambda_{e} \simeq \sqrt{\Delta \mathcal{M}_{n u c}}\right)$ the main conclusions do not depend on this specific choice.
In the following analysis we will use the currently most stringent limit on double beta decay

$$
T_{1 / 2}\left({ }^{76} G e\right) \geq 1.1 \times 10^{25} y s
$$

found by the Heidelberg-Moscow experiment [i] $\left.\overline{1}_{1}\right]$.

The experimental constraint eq. (3.1) together with the theoretical decay rate eq. (12.18 defines an excluded area in a complex multi dimensional parameter space consisting of the $\not R_{p}$ parameters as well as a number of MSSM parameters.

Let us first compare the short-range contributions with the long-range (light) neutrino exchange. All of them are of the same order in RPV parameters, i.e. proportional to $\Lambda_{1}^{2}$. By naively comparing the corresponding diagrams one might think that they are of equal importance. Closer inspection, however, reveals that this is not the case, and that the short-range contributions are suppressed compared to the longrange ones. This suppression is due to nuclear physics effects. For heavy particle exchange the two quarks interacting have to come very close together, where the strong repulsive part of the nucleon-nucleon interaction is important. For point-like nucleons this contribution would be zero, so that the corresponding nuclear matrix elements become non-zero only due to the finite nucleon size. A naive estimate of the size of the short-range contributions relative to the neutrino mass contribution can be given by comparing the typical momentum scale of the nucleons, order $p_{F}$, with the typical mass scale of SUSY particles $m_{S U S Y}$ and thus is expected to be $p_{F} / m_{S U S Y} \sim 10^{-3}$.

Fig. 2 then shows the limits on the parameters $\epsilon_{1}$, for $\omega_{1}=0$, assuming $\tan \beta=1$ as a function of the MSSM parameters $\left(M_{2}, \mu\right)$. Limits typically of the order of a few hundred $\mathrm{keV}$ are found, choosing $M_{2}$ and $\mu$ of the order of up to a few hundred GeV. Note, however, that the limit on $\epsilon_{1}$ depends rather strongly on the choice of $\tan \beta$, and detoriates proportional to $\tan \beta$ for large values of this parameter. 


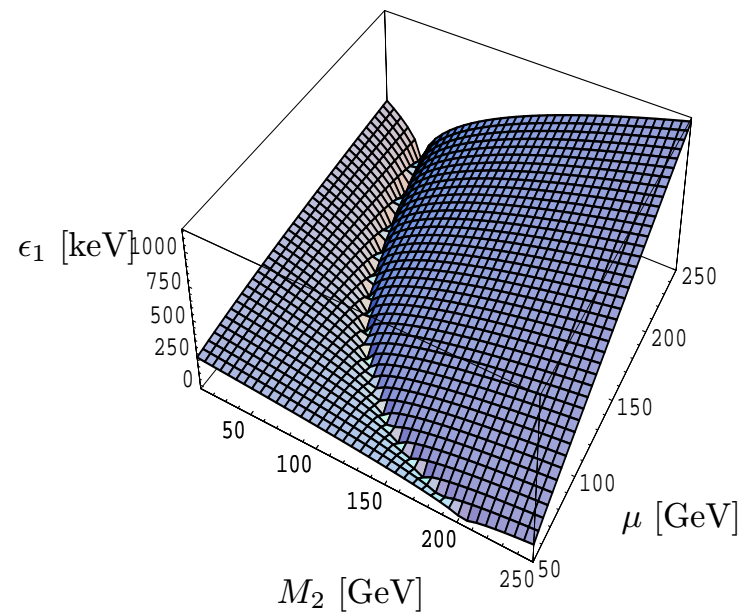

Figure 2: Upper limit on the individual parameter $\epsilon_{1}$ for $\tan \beta=1$ and $\omega_{i}=0$ as a function of $\left(M_{2}, \mu\right)$.

While figure 2 shows, that for typical ranges of $M_{2}$ and $\mu$ the upper bound on $\epsilon_{1}$ is of the order of a few $100 \mathrm{keV}$, the limits in figure 2 are not completely realistic, since they make the rather unreasonable assumption $\omega_{1}=0$. It is more realistic to plot the excluded ranges of parameters in the $\left(\epsilon_{1}, \omega_{1}\right)$ plane, for different choices of $M_{2}$ and $\mu$. The results are shown in figure 3 .

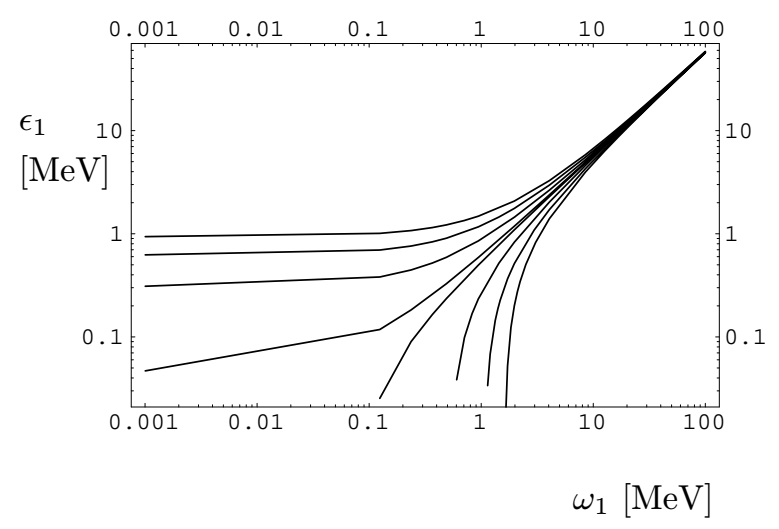

Figure 3.a: Upper limits on $\left(\epsilon_{1}, \omega_{1}\right)$ assuming $\left\langle m_{\nu}\right\rangle \leq 0.5 \mathrm{eV}, \tan \beta=1$ and $\mu=100 \mathrm{GeV}$, for different values of $M_{2}, M_{2}=100,200,500,1000$ GeV. Note that the allowed range is always in between two lines of constant $M_{2}$.

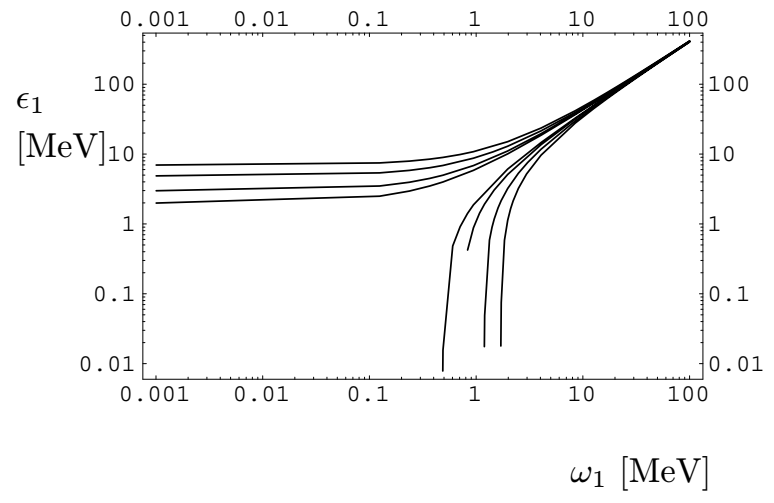

Figure 3.b: Same as figure 3.a, but for $\tan \beta=$ 10.

Figure 3 shows, that assuming either $\epsilon_{1}$ or $\omega_{1}$ to be equal to zero, one gets the correct order of magnitude of constraint on the other quantity, except in the narrow region where both are approximately aligned to give $\Lambda_{1}=0 .{ }^{3}$

Comparing figure 3.a with fig. $3 . \mathrm{b}$ it is also seen, that the limit on $\epsilon$ depends on the choice of $\tan (\beta)$, whereas the limit on $\omega_{1}$ does not. As long as $M_{2}$ and $\mu$ are in a realistic range, say below $1 \mathrm{TeV}$, typical limits on $\omega_{1}$ of the order of a few $\mathrm{MeV}$ are obtained.

\section{Conclusion}

I have discussed the contributions of (bilinear) Rparity breaking supersymmetry to neutrinoless double beta decay. This model mimics closely the case of spontaneous breaking of R-parity. It is found that $\beta \beta_{0 \nu}$ decay constrains only a subset of the possible bilinear parameters, namely $\epsilon_{1}$ and the sneutrino VEV of the first generation $\omega_{1}$. For the first generation $\not R_{p}$ parameters $\beta \beta_{0 \nu}$ decay provides very stringent limits, typically of the order of a few hundred $\mathrm{keV}$ up to a few MeV. With these limits at hand it seems rather hopeless to search for RPV in the first generation at colliders.

\footnotetext{
${ }^{3}$ In the tree-level calculation presented here, the double beta decay amplitude is proportional to $\Lambda_{1}^{2}$. However, once 1-loop corrections are taken into account, this strict proportionality is broken.
} 
${ }^{4}$ From this point of view a possible interpretation of HERA events in terms of $R_{p}$ interactions would be rather unlikely. However, it is important to stress that, even though rather stringent constraints on the magnitude of the first generation $R_{p}$ parameters have been found, these do not limit in any way the attainable magnitudes of R-parity breaking signatures expected at colliders, since the latter involve mainly the third generation, i.e. they involve $\tau$ 's or $\nu_{\tau}$ 's.

In the present study it is found that the main origin $\beta \beta_{0 \nu}$ decay in this model is the mass mechanism, other contributions are practically irrelevant.

\section{Acknowledgement}

I am grateful to S.G. Kovalenko for useful comments and to J.W.F. Valle for his contributions to the work presented here. This work was supported by the Spanish DGICYT under grant PB95-1077 and by the European Union's TMR program under grants ERBFMRXCT960090 and ERBFMBICT983000.

\section{References}

[1] For a review on the MSSM, see for example: H.E. Haber and G.L.Kane, Phys. Rep. 117 (1985) 75

[2] Hall L. and Suzuki M., Nucl. Phys. B231 419 (1984)

[3] G. G. Ross, J. W. F. Valle. Phys.Lett. 151B 375 (1985); John Ellis, G. Gelmini, C. Jarlskog, G.G. Ross, J. W. F. Valle, Phys.Lett. 150B 142 (1985)

[4] For a recent analysis of atmospheric neutrino data see M. C. Gonzalez-Garcia, H. Nunokawa, O. L. G. Peres, J. W. F. Valle, [hep-ph/9807305]; M. C. Gonzalez-Garcia, H. Nunokawa, O. L. G. Peres, T. Stanev, J. W. F. Valle, Phys. Rev. D58, 033004, (1998) [hep$\mathrm{ph} / 9801368]$.

[5] For a recent analysis of the solar neutrino data see J.N. Bahcall, P.I. Krastev, A.Yu. Smirnov, Phys. Rev. D58, 096016, (1998).

\footnotetext{
${ }^{4}$ However, there is one exception from this general statement. The lightest neutralino (lightest of the 4 heavy states) is unstable in this model whenever at least one generation has non-vanishing RPV parameters. The question whether or not the lightest neutralino decays inside the detector for such small values of RPV parameters or not has to be investigated.
}

[6] For a recent review on the status of neutrino physics see, e.g. J. W. F. Valle, hep-ph/9809234

[7] J. Schechter and J.W.F. Valle, Phys.Rev. D 25 (1982) 2951

[8] M. Hirsch, H.V. Klapdor-Kleingrothaus and S.G. Kovalenko, Phys. Rev. Lett. 75 (1995) 17; Phys. Rev. D53 (1996) 1329; Phys. Lett. B372 (1996) 181, erratum B381 (1996) 488

[9] A. Faessler, S.G. Kovalenko, F. Simkovic and J. Schwieger, Phys. Rev. Lett. 78 (1997) 183

[10] A. Faessler, S. Kovalenko, F. Simkovic, Phys.Rev. D57 (1998) 055004 [hep-ph/9712535]

[11] M. Hirsch and J.W.F. Valle, hep-ph/9812463 and Nucl.Phys. B (1999), in press

[12] M.A. Díaz, J.C. Romão, and J.W.F. Valle, Nucl.Phys. B524 23-40 (1998), [hep$\mathrm{ph} / 9706315]$

[13] M. Hirsch, H.V. Klapdor-Kleingrothaus and S.G. Kovalenko, Phys. Lett. B372 (1996) 181; Erratum Phys.Lett. B381 (1996) 488

[14] M. Doi, T. Kotani, E. Takasugi, Progr. Theor. Phys. Suppl. 83 (1985) 1

[15] K. Muto, E. Bender and H.V. Klapdor, Z. Phys. A334 (1989) 177, 187

[16] M. Hirsch, H.V. Klapdor-Kleingrothaus and O. Panella, Phys. Lett. B374 (1996) 7

[17] L. Baudis et al., Phys. Lett. B407 (1997) 219 